\title{
Knockdown of receptor tyrosine kinase-like orphan receptor 2 inhibits cell proliferation and colony formation in osteosarcoma cells by inducing arrest in cell cycle progression
}

\author{
JIANJUN HUANG ${ }^{1}$, YING SHI ${ }^{2}, \mathrm{HUI} \mathrm{LI}^{3}$, DUNYONG TAN ${ }^{3}$, MEISONGZHU YANG ${ }^{2}$ and XIANG WU ${ }^{1}$ \\ ${ }^{1}$ The Second Department of Orthopedics, The First Affiliated Hospital of Jishou University; \\ ${ }^{2}$ Teaching and Research Department of Pathology and Pathophysiology; ${ }^{3}$ Department of Immunology and Microbiology, \\ Medical School of Jishou University, Jishou, Hunan 416000, P.R. China
}

Received October 11, 2014; Accepted August 13, 2015

DOI: $10.3892 / \mathrm{ol} .2015 .3797$

\begin{abstract}
Osteosarcoma (OS) is the most common malignant tumor of the bone, with a high mortality rate and poor prognosis. Receptor tyrosine kinase-like orphan receptor 2 (ROR2) has been reported to be dysregulated in human malignancies. More recently, ROR2 has been demonstrated to promote OS cell migration and invasion. However, the role of ROR2 in the regulation of OS cell proliferation, as well as the underlying molecular mechanism, remains unclear. The present study aimed to investigate the underlying mechanism of ROR2 in osteosarcoma growth. Reverse transcription-quantitative polymerase chain reaction analysis and western blot analysis were used to examine the mRNA and protein expression. MTT assay, colony formation assay and cell cycle analysis were conducted to explore the function of ROR2 in osteosarcoma cells. In the present study, the expression of ROR2 was found to be frequently upregulated in OS tissues compared with matched adjacent normal tissues. It was also upregulated in the OS cell lines Saos-2, MG-63 and U-2 OS, relative to normal osteoblast hFOB 1.19 cells. Knockdown of ROR2 expression by transfection with ROR2-specific siRNA markedly inhibited the proliferation and colony formation of OS cells. Data from the cell cycle distribution assay revealed an accumulation of ROR2-knockdown cells in the G0/G1 phase, indicating that knockdown of ROR2 leads to an arrest in cell cycle progression. Mechanistic investigation revealed that the protein levels of c-myc, a target gene of the Wnt signaling, as well as cyclin D1, cyclin E and cyclin-dependent kinase 4 were markedly reduced in the ROR2-knockdown OS cells, suggesting
\end{abstract}

Correspondence to: Professor Jianjun Huang, The Second Department of Orthopedics, The First Affiliated Hospital of Jishou University, 120 Renmin Middle Road, Jishou, Hunan 416000, P.R. China

E-mail: jishouhuangjianjun@163.com

Key words: osteosarcoma, receptor tyrosine kinase-like orphan receptor 2, proliferation, colony formation, cell cycle progression that the inhibitory effect of ROR 2 knockdown on OS cell proliferation is associated with the Wnt signaling pathway. In summary, the current study indicates an important role for ROR2 in the proliferation of OS cells. Therefore, ROR2 may be a promising therapeutic target in OS.

\section{Introduction}

Osteosarcoma (OS) is the most common malignant tumor of the bone, with a peak incidence in young children and adolescents. The incidence of osteosarcoma is $0.20-0.35 / 100,000$ individuals (1). OS is associated with a high rate of mortality: The five year overall survival is $75-77 \%$ for the primary non-metastatic osteosarcoma, and no more than $20 \%$ for metastatic osteosarcoma $(2,3)$. Despite the use of surgical excision combined with chemotherapy and radiotherapy, the median survival rate of OS remains poor (4). It has been demonstrated that dysregulation of oncogenes or tumor suppressor genes is involved in the development and progression of OS (5). Establishing novel therapeutic targets is urgently required for the diagnosis and treatment of OS.

Receptor tyrosine kinase (RTK)-like orphan receptor 2 (ROR2) belongs to the RTK family, which is important in the regulation of numerous cellular biological processes, including proliferation, apoptosis, differentiation, adhesion and migration (6-8). ROR2 is expressed in heart, brain and lung tissue, and is also involved in the development of the nervous system and bones (9-11). It has been hypothesized that ROR2 is involved in the early formation of chondrocytes as well as the development of cartilage and growth plates (12). In addition, mutations in the ROR2 gene can cause the autosomal recessive form of Robinow syndrome, a rare disorder that is characterized by skeletal dysplasia, limb bone shortening, segmental defects of the spine, brachydactyly and facial abnormalities (13).

It has been demonstrated that ROR2 is frequently downregulated in a number of common types of malignancy, including esophageal, nasopharyngeal, gastric, colorectal, hepatocellular, lung and breast cancers $(14,15)$. ROR2 acts as a tumor suppressor by inhibiting the epithelial-mesenchymal transition and tumor cell stemness through repressing 
$\beta$-catenin and AKT signaling (16). Recently, it has been indicated that Wnt5a/ROR2 signaling may be associated with OS severity, and plays a promotive role in the regulation of OS cell migration and invasion (17-19). However, the precise roles of ROR2 in the regulation of OS cell proliferation, as well as the underlying mechanism, have not previously been reported.

The present study aimed to explore the role of ROR2 in the regulation of OS cell proliferation and to investigate the underlying molecular mechanisms.

\section{Materials and methods}

Reagents and materials. RPMI 1640 medium, fetal bovine serum (FBS), Trizol Reagent and Lipofectamine 2000 were purchased from Life Technologies (Grand Island, NY, USA). Dimethyl sulfoxide (DMSO) and MTT were purchased from Sigma-Aldrich (St. Louis, MO, USA). PrimeScript RT Reagent Kit and SYBR Premix Ex Taq II were purchased from Takara Biotechnology Co., Ltd., (Dalian, China).ROR2-specific small interfering RNA (siRNA) and non-specific siRNA were generated from Nlunbio (Changsha, China). A Pierce Enhanced Chemiluminescence (ECL) kit was purchased from Thermo Fisher Scientific (Rockford, IL, USA). Transwell inserts were purchased from BD Biosciences (San Jose, CA, USA). Mouse anti-ROR2 monoclonal antibody, mouse anti-GAPDH monoclonal antibody and rabbit anti-mouse secondary antibody were purchased from Abcam (Cambridge, UK).

Tissue specimen collection. All protocols in this study were approved by the Ethics Committee of Jishou University (Jishou, China). A total of 18 OS tissues as well as their matched adjacent normal tissues were collected at The Second Department of Orthopedics of the First Affiliated Hospital of Jishou University between December 2012 and December 2013. The 18 cases included 7 female and 11 male who ranged in age between 25 and 64 years, with a mean of 48.5 years. All patients received neither radiation therapy nor chemotherapy before surgical resection. Among all OS patients, 2 cases were classified as grade I, 7 grade II, 6 grade III, and 3 grade IV. Written informed consent was obtained from all patients. Tissues were immediately snap-frozen in liquid nitrogen following surgical removal, and stored at $-70^{\circ} \mathrm{C}$ until use.

Cell culture. The human OS cell lines Saos-2, MG-63 and U-2 OS and the human osteoblast cell line hFOB 1.19 were obtained from the American Type Culture Collection (Manassas, VA, USA). Cells were cultured in RPMI 1640 medium supplemented with $10 \%$ FBS, $100 \mathrm{U} / \mathrm{ml}$ penicillin and $100 \mathrm{mg} / \mathrm{ml}$ streptomycin (Sigma-Aldrich) in a humidified atmosphere of $5 \% \mathrm{CO}_{2}$ at $37^{\circ} \mathrm{C}$.

Reverse transcription (RT)-quantitative polymerase chain reaction $(q P C R)$ analysis. Trizol Reagent was used to extract total RNA from tissues or cells, in accordance with the manufacturer's instructions, and a total of 800 ng RNA was subsequently reverse transcribed into cDNA using a PrimeScript RT Reagent Kit, according to the manufacturer's protocol. Reverse transcription was performed at $16^{\circ} \mathrm{C}$ for $30 \mathrm{~min}$, followed by an incubation at $42^{\circ} \mathrm{C}$ for $30 \mathrm{~min}$ and enzyme inactivation at $85^{\circ} \mathrm{C}$ for $5 \mathrm{~min}$. The mRNA expression level was determined using SYBR Premix Ex Taq II, on ABI 7500 thermocycler (Thermo Fisher Scientic, Inc.), in accordance with the manufacturer's instructions. The reaction conditions were as follows: $95^{\circ} \mathrm{C}$ for $5 \mathrm{~min}$, followed by 40 cycles of denaturation at $95^{\circ} \mathrm{C}$ for $15 \mathrm{sec}$ and an annealing/elongation step at $60^{\circ} \mathrm{C}$ for $30 \mathrm{sec}$. The specific primer pairs are as follows: ROR2 sense, 5'-GTGCGGTGGCTAAAGAATGAT-3; ROR2 antisense, 5'-ATTCGCAGTCGTGAACCATATT-3'; GAPDH (internal reference) sense, 5'-ACAACTTTGGTATCGTGGAAGG-3'; and GAPDH antisense, 5'-GCCATCACGCCACAGTTTC-3'. Independent experiments were repeated three times. The relative expression of ROR 2 mRNA was analyzed using the $2^{-\Delta \Delta \mathrm{Ct}}$ method.

Transfection. Cells were cultured to $70 \%$ confluence and resuspended in serum-free medium. Lipofectamine 2000 was used to transfect cells with ROR2 siRNA or with non-specific siRNA as a negative control (NC). In brief, serum-free medium was used to dilute Lipofectamine 2000 and siRNA, and the diluted Lipofectamine 2000 was then added into the diluted siRNA. Following incubation for $20 \mathrm{~min}$ at room temperature, the mixture was added to the cell suspension. The cells and transfection reagents were incubated at $37^{\circ} \mathrm{C}$ in an atmosphere of $5 \% \mathrm{CO}_{2}$ for $6 \mathrm{~h}$, before the medium was replaced by normal serum-containing medium. After transfection for $48 \mathrm{~h}$, the following assays were performed.

Proliferation assay. An MTT assay was performed to determine cell proliferation. In brief, $10^{4}$ cells per well were plated in a 96-well plate, and incubated for $6,12,24$ or $48 \mathrm{~h}$ at $37^{\circ} \mathrm{C}$ in an atmosphere of $5 \% \mathrm{CO}_{2}$. Subsequently, $10 \mu \mathrm{l}$ of MTT solution $(5 \mathrm{mg} / \mathrm{ml})$ was added to each well and incubated for $4 \mathrm{~h}$ at $37^{\circ} \mathrm{C}$ in $5 \% \mathrm{CO}_{2}$. The supernatant was then removed, and $100 \mu 1$ of DMSO was added to dissolve the precipitate. The absorbance [optical density (OD)] was detected at $492 \mathrm{~nm}$ with a 680 microplate reader (Bio-Rad Laboratories, Inc.).

Colony formation assay. For all three groups, 150 cells in $3 \mathrm{ml}$ complete medium were added to each well of a 6 -well plate, which were then incubated at $37^{\circ} \mathrm{C}$ in $5 \% \mathrm{CO}_{2}$ for 14 days. At the end of this period, the cells were washed and stained with Giemsa (Sigma-Aldrich). Colonies composed of $\geq 50$ cells were then counted.

Cell cycle analysis. Cells were collected in $1 \mathrm{X}$ phosphate-buffered saline (PBS) and fixed in $70 \%$ ethanol overnight at $-20^{\circ} \mathrm{C}$. The cells were subsequently pelleted at $200 \mathrm{x} \mathrm{g}$ for $5 \mathrm{~min}$, washed in $1 \mathrm{X}$ PBS, and pelleted again at $200 \mathrm{x} \mathrm{g}$ for $5 \mathrm{~min}$. Cells were resuspended in $300 \mu \mathrm{l}$ propidium iodide (PI; Sigma-Aldrich) and incubated at room temperature for 30 min. DNA content analyses were conducted using a C6 flow cytometer (Beckman Coulter, Brea, CA, USA).

Western blotting. Western blotting was performed to determine relative protein expression. In brief, tissues or cells were solubilized in cold radioimmunoprecipitation assay lysis buffer (Sbjbio, Nanjing, China). Proteins were separated using 10\% SDS-PAGE (Sbjbio), and transferred onto a polyvinylidene fluoride (PVDF) membrane (Thermo Fisher Scientific), which was then incubated with mouse monoclonal anti-human ROR2 
A

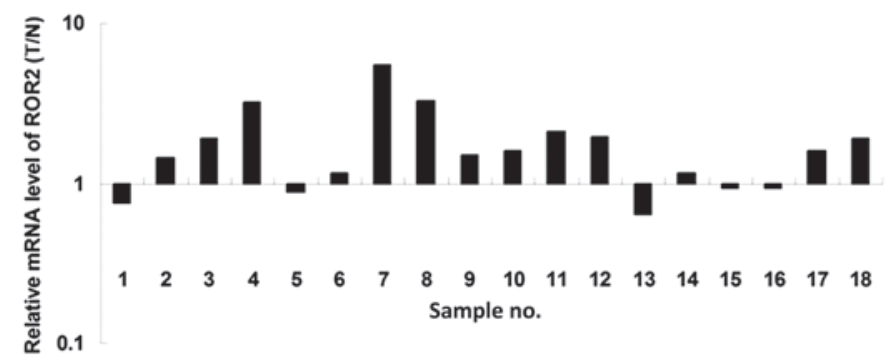

C

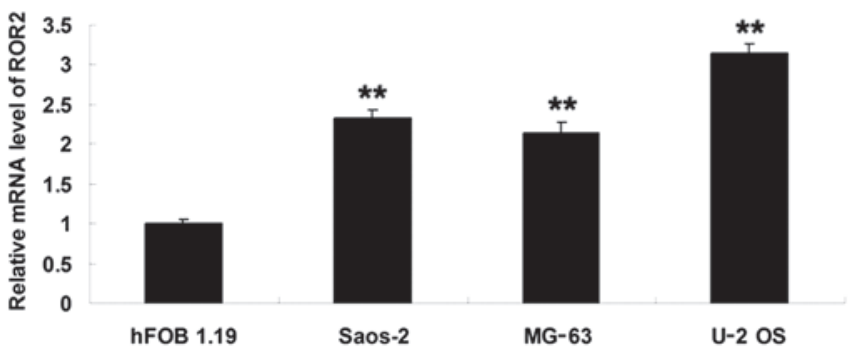

B

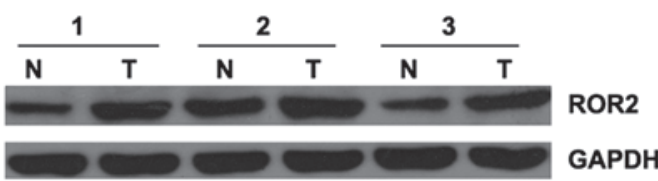

D
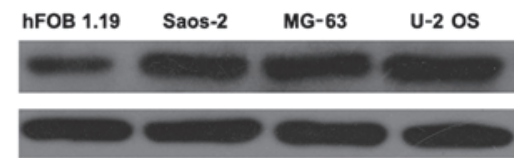

ROR2

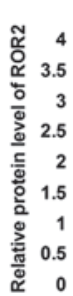

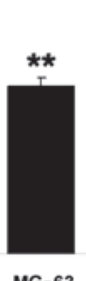

MG-63
GAPDH

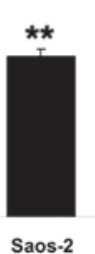

Figure 1. ROR2 expression in OS. (A) The expression of ROR2 mRNA was examined using RT-qPCR in 18 samples of OS tissue and their matched adjacent normal tissues. (B) ROR2 protein expression was examined by western blotting in OS tissues and matched adjacent normal tissues. (C) The mRNA expression of ROR2 was examined by using RT-qPCR in the human OS cell lines Saos-2, MG-63 and U-2 OS, as well as in the human osteoblast cell line hFOB 1.19 ( ${ }^{* *} \mathrm{P}<0.01$ vs. hFOB 1.19). (D) The protein expression of ROR2 was examined by western blotting in the human OS cell lines Saos-2, MG-63 and U-2 OS, as well as in the human osteoblast cell line hFOB 1.19 ("* $\mathrm{P}<0.01 \mathrm{vs.} \mathrm{hFOB} \mathrm{1.19).} \mathrm{ROR2,} \mathrm{receptor} \mathrm{tyrosine} \mathrm{kinase-like} \mathrm{orphan} \mathrm{receptor} \mathrm{2;} \mathrm{RT-qPCR,} \mathrm{reverse}$ transcription-quantitative polymerase chain reaction; OS, osteosarcoma; T, tumor tissue; N, normal (tumor-adjacent) tissue.

(ab201962, 1:100), monoclonal mouse anti-human cyclin D1 (ab187892, 1:200), monoclonal mouse anti-human cyclin E (ab3927, 1:50), monoclonal mouse anti-human CDK4 (ab75511, 1:200), monoclonal mouse anti-human $c$-myc (ab56, 1:200) and monoclonal mouse anti-human GAPDH (ab8245, 1:200, used as internal control) at room temperature for $3 \mathrm{~h}$. Following three washes in PBS-Tween 20, the PVDF membrane was incubated with the rabbit anti mouse secondary antibody (ab175743, 1:10,000) at room temperature for $1 \mathrm{~h}$. Chemiluminescence detection was performed using an ECL kit and the relative protein expression was analyzed using Image-Pro Plus software version 6.0 (Media Cybernetics, Inc., Rockville, MD, USA), represented as the ROR2 density ratio relative to that of GAPDH.

Statistical analysis. All data is represented as the mean \pm standard deviation of at least triplicate samples. A Student's $t$-test or $\chi^{2}$ test was used to statistically analyze data with SPSS software version 17 (SPSS, Inc., Chicago, IL, USA). $\mathrm{P}<0.05$ was considered to indicate statistically significant differences.

\section{Results}

ROR2 expression was significantly upregulated in OS tissues and cell lines. To investigate the role of ROR 2 in OS, its expression levels were examined in OS tissue specimens as well as their matched adjacent normal tissues by RT-qPCR. As shown in Fig. 1A, mRNA expression was significantly upregulated in OS tissues compared with that of matched adjacent normal tissue samples. Similarly, the results of western blotting revealed that ROR2 protein expression was also upregulated in OS tissues (Fig. 1B). To confirm this, mRNA and protein expression of ROR2 was also evaluated in three OS cell lines, with the human osteoblast cell line hFOB 1.19 used as a control. As shown in Fig. 1C and D, the expression level of ROR2 was markedly increased in OS cell lines compared with that in hFOB 1.19 cells. These findings indicate that ROR2 may be involved in the development of OS.

siRNA-induced ROR2 downregulation inhibits OS cell proliferation. As U-2 OS cells demonstrated the highest ROR2 mRNA and protein expression, this cell line was used in the subsequent in vitro experiments. ROR2-specific siRNA was transfected into U-2 OS cells to knockdown ROR2 expression. As shown in Fig. 2A and B, the relative mRNA and protein expression of ROR2 in the ROR2 siRNA group was markedly decreased when compared with that of the Control (untransfected) group, while the difference between the NC and Control groups was not statistically significant. These data indicated that the expression of ROR2 in U-2 OS cells was successfully downregulated. Subsequently, MTT assays were conducted to investigate the effect of ROR2 downregulation on U-2 OS cell proliferation. As shown in Fig. 2C, the OD value of U-2 OS cells in the ROR2 siRNA group was significantly lower than that of the Control and NC groups $(\mathrm{P}<0.01)$, suggesting that inhibition of ROR 2 expression markedly inhibited the proliferation of U-2 OS cells.

Knockdown of ROR2 expression inhibits colony formation of $O S$ cells. A colony formation assay was performed to investigate the effect of ROR2 downregulation on the colony-forming capacity of U-2 OS cells. As shown in Fig. 3, the colony-forming capacity of U-2 OS cells transfected with ROR2 siRNA was markedly decreased compared with that of 

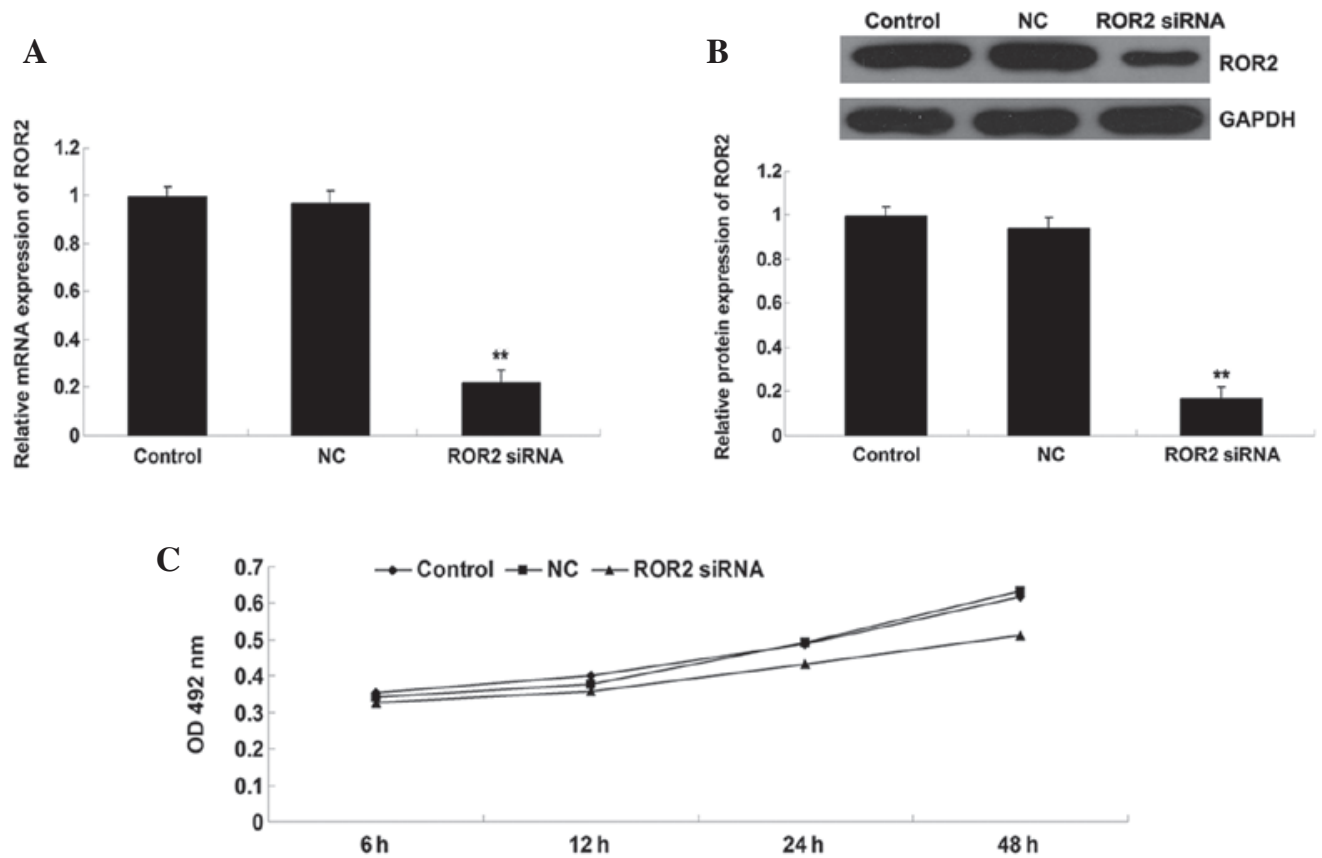

Figure 2. (A) The mRNA expression of ROR2 was examined by RT-qPCR in U-2 OS cells with or without transfection with ROR2-specific siRNA $\left({ }^{* *} \mathrm{P}<0.01\right.$ vs. control). (B) The protein expression of ROR2 was examined by western blotting in U-2 OS cells with or without transfection with ROR2-specific siRNA ( ${ }^{* *} \mathrm{P}<0.01$ vs. control). (C) MTT assays were performed at $6,12,24$ or $48 \mathrm{~h}$ after plating to determine the proliferation capacity of U-2 OS cells with or without transfection with ROR2-specific siRNA ( ${ }^{* * *} \mathrm{P}<0.01$ vs. control). Control, untransfected U-2 OS cells; NC (negative control), U-2 OS cells transfected with non-specific siRNA. ROR2, receptor tyrosine kinase-like orphan receptor 2; RT-qPCR, reverse transcription-quantitative polymerase chain reaction; siRNA, small interfering RNA; OD optical density.

the Control and $\mathrm{NC}$ groups, indicating that downregulation of ROR2 expression significantly suppressed the colony-forming capacity of U-2 OS cells.

Downregulation of ROR2 expression results in arrest of cell cycle progression of OS cells. To further investigate the molecular mechanism of ROR2 in OS, cell cycle progression, which is closely associated with cell proliferation and colony formation, was analyzed in U-2 OS cells in each group. Data from the cell cycle distribution assay revealed an accumulation of ROR2-knockdown U-2 OS cells at G1 phase, and a decrease in S and G2 phases compared with the control groups (Fig. 4). These findings indicated that knockdown of ROR2 induced an arrest in cell cycle progression, which may be the primary reason for the reduced proliferative and colony-forming capacities of ROR2-knockdown U-2 OS cells.

ROR2 knockdown leads to downregulation of cell cycle proteins and c-myc. Consistent with the aforementioned data, the protein expression levels of cyclin D1, cyclin E, and cyclin-dependent kinase 4 (CDK4), which are involved in the cell cycle G1-S phase transition, were significantly reduced in ROR2-knockdown OS cells (Fig. 5A). In addition, c-myc, which has been demonstrated to play a crucial role in cell proliferation, was also found to have reduced expression in ROR2-knockdown OS cells compared with the control groups (Fig. 5B).

\section{Discussion}

ROR2, a member of RTK family, is a type I transmembrane protein that belongs to the RTK-like orphan receptor subfamily of cell surface receptors (20). ROR2 is associated with the
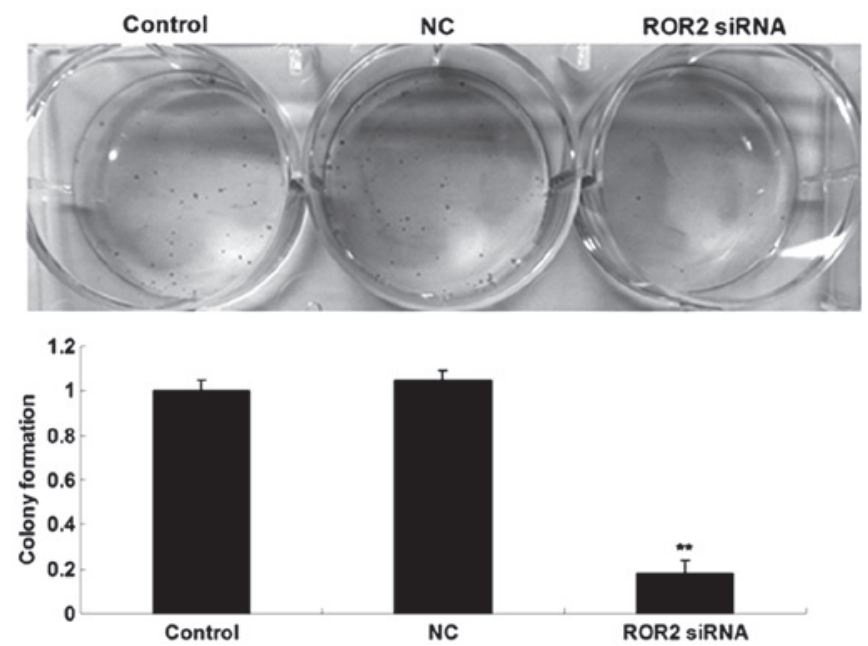

Figure 3. Colony formation assay was performed in U-2 OS cells with or without transfection with ROR2-specific siRNA. Control, untransfected U-2 OS cells; NC (negative control), U-2 OS cells transfected with non-specific siRNA. ${ }^{* *} \mathrm{P}<0.01$ vs. Control.

development and progression of numerous types of human cancer, including OS $(14,15)$. ROR2 has been suggested to play a role in the regulation of OS cell migration and invasion (21). However, the precise role of ROR2 in the regulation of OS cell proliferation, as well as its underlying mechanism, remains largely unclear. In the current study, the expression of ROR2 was found to be significantly increased in OS tissues and cell lines, and knockdown of ROR2 expression by transfection with ROR2-specific siRNA markedly inhibited the proliferation and colony formation of OS cells. These findings suggest 

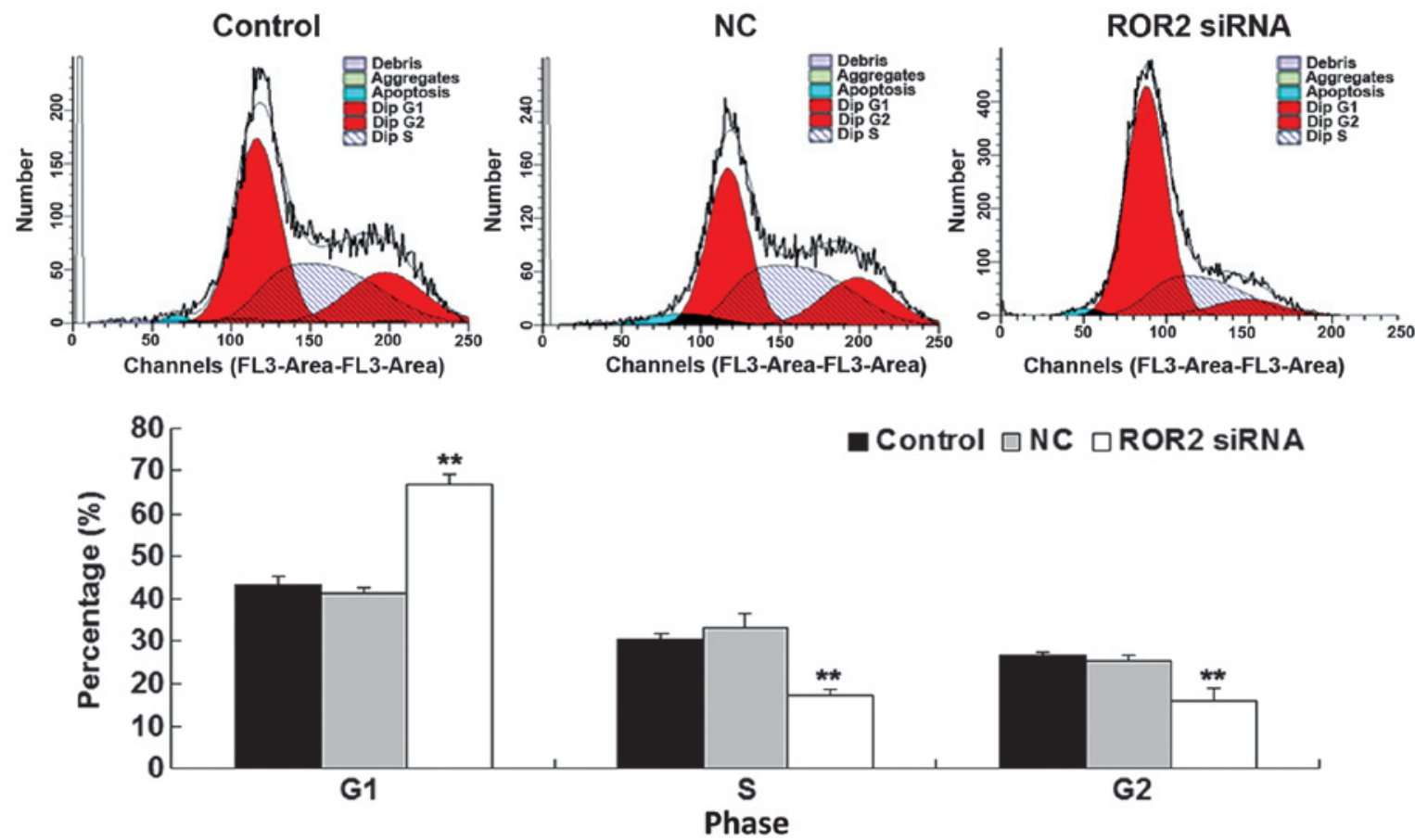

Figure 4. A cell cycle distribution assay was performed in U-2 OS cells with or without transfection with ROR2-specific siRNA. ${ }^{* *} \mathrm{P}<0.01$ vs. control. ROR2, receptor tyrosine kinase-like orphan receptor 2; Control, untransfected U-2 OS cells; NC (negative control), U-2 OS cells transfected with non-specific siRNA.
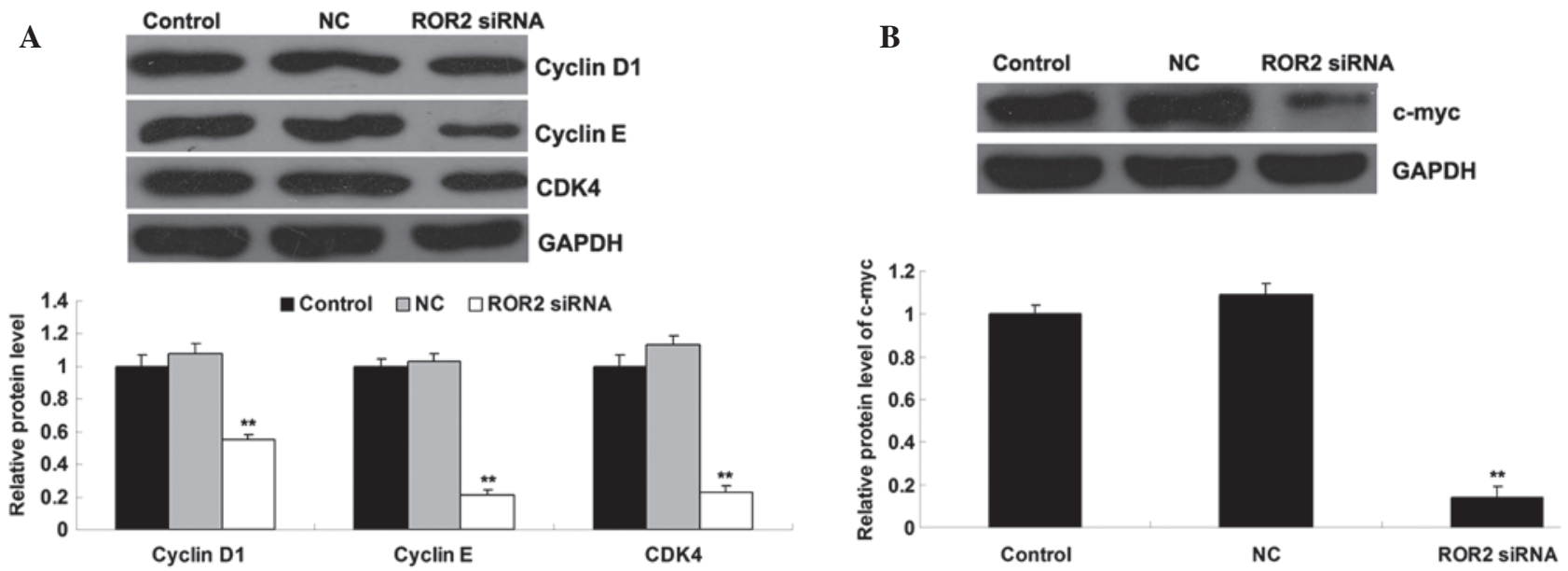

Figure 5. (A) Western blotting was performed to examine the protein expression levels of cyclin D1, cyclin E and CDK4 in U-2 OS cells with or without transfection with ROR2-specific siRNA. GAPDH was used as an internal reference. (B) Western blotting assay was performed to examine the protein expression level of c-myc in U-2 OS cells with or without transfection with ROR2-specific siRNA. GAPDH was used as an internal reference. ${ }^{* *} \mathrm{P}<0.01 \mathrm{vs}$. control. CDK4, cyclin-dependent kinase 4; ROR2, receptor tyrosine kinase-like orphan receptor 2; Control, untransfected U-2 OS cells; NC (negative control), U-2 OS cells transfected with non-specific siRNA.

that ROR2 plays a promotive role in the regulation of OS cell proliferation, and that knockdown of ROR2 expression may be effective for inhibiting the development and growth of OS.

As the downregulation of cell proliferation and colony formation induced by ROR 2 knockdown may be due to the inhibition of cell cycle progression, a cell cycle distribution assay was also conducted. These findings revealed an accumulation of ROR2-knockdown OS cells in G0/G1 phase, and a decrease in $\mathrm{S}$ and $\mathrm{G} 2 / \mathrm{M}$ phases, indicating that knockdown of ROR2 leads to an arrest in cell cycle progression of OS cells. Further findings revealed that the expression levels of cyclin D1, cyclin E and CDK4 were significantly reduced in
ROR2-knockdown OS cells. These molecules are involved in the G0/G1-S transition of the cell cycle, and thus the regulation of cell proliferation and colony formation (22).

Furthermore, ROR2 is involved in the Wnt signaling pathway (23). Wnt signaling is important in normal embryonic pattern formation and cell differentiation, as well as tumorigenesis $(9,24)$, and may therefore act as potential diagnostic or therapeutic target for human malignancies. As a novel Wnt receptor, ROR2 provides the potential to target the non-canonical Wnt pathway for cancer treatments. Notably, ROR2 appears to possess dual roles as an oncogene or tumor suppressor depending on tumor type (25). For 
instance, the protein expression of ROR2 was reported to be significantly decreased in hepatocellular carcinoma (HCC) tissues compared with adjacent non-tumorous tissues, and loss of ROR2 expression was associated with poor prognosis, suggesting that ROR2 may act as a tumor suppressor in HCC (26). By contrast, ROR2 was suggested to play an oncogenic role in certain other cancers, including melanoma, renal cell cancer and oral squamous cell carcinoma (27-29). ROR 2 promotes the proliferation and migration of renal cell carcinoma cells in vitro and in vivo (30).

The role of Wnt/ROR2 signaling in the regulation of OS development has been gradually elucidated. Wnt5b has been identified as a ligand of ROR2, and the physiological interaction of ROR2 and Wnt5b may enhance OS cell migration, suggesting that Wnt/ROR2 signaling may be involved in OS metastasis (31). Furthermore, Wnt/ROR2 signaling was found to promote OS cell invasion, at least in part, through the activation of a Src-family protein tyrosine kinase as well as upregulation of matrix metalloproteinase-13 expression (19,21). In addition, Lu et al (17) reported that ROR2 and Wnt5a were significantly upregulated in OS tissues, and their expression levels were correlated with Enneking surgical stage and tumor metastasis. In the current study, cyclin D1, a target gene of Wnt signaling (32), was markedly downregulated following knockdown of ROR2 in OS cells. In addition, c-myc, another target gene of Wnt signaling that has been demonstrated to participate in the regulation of cell proliferation (33), was found to have reduced expression in ROR2-knockdown OS cells. Taken together, these findings suggest that the inhibitory effect of ROR2 knockdown on OS cell proliferation may be associated with the Wnt signaling pathway.

In summary, the present study revealed that the expression level of ROR2 was significantly increased in OS tissues and cell lines. We hypothesize that ROR2 is able to promote the proliferation of OS cells through the regulation of cell cycle progression, and that the Wnt signaling pathway is involved in ROR2-mediated OS cell proliferation. Therefore, ROR 2 may become a potential molecular target for the treatment of OS.

\section{Acknowledgements}

This study was supported by the Scientific Research Project of Education Department of Hunan Province (no. 11C1035), Natural Science Fund Project of Hunan Province (no. 12JJ6074), Research Project of Health Department of Hunan Province (no. B2013-160) and the Introduction of Talent Research Project of Jishou University (no. jsdxrcyjkyxm201110).

\section{References}

1. Valery PC, Laversanne M and Bray F: Bone cancer incidence by morphological subtype: A global assessment. Cancer Causes Control 26: 1127-1139, 2015.

2. Ferrari S, Smeland S, Mercuri M, Bertoni F, Longhi A, Ruggieri $P$, Alvegard TA, Picci P, Capanna R, Bernini G, et al; Italian and Scandinavian Sarcoma Groups: Neoadjuvant chemotherapy with high-dose Ifosfamide, high-dose methotrexate, cisplatin, and doxorubicin for patients with localized osteosarcoma of the extremity: A joint study by the Italian and Scandinavian Sarcoma Groups. J Clin Oncol 23: 8845-8852, 2005.
3. Mialou V1,Philip T, Kalifa C,Perol D, Gentet JC,Marec-Berard P, Pacquement H, Chastagner P, Defaschelles AS and Hartmann O: Metastatic osteosarcoma at diagnosis: prognostic factors and long-term outcome - the French pediatric experience. Cancer 104: 1100-1109, 2005.

4. Thompson LD: Osteosarcoma. Ear Nose Throat J 92: 288-290, 2013.

5. PosthumaDeBoer J, Witlox MA, Kaspers GJ and van Royen BJ: Molecular alterations as target for therapy in metastatic osteosarcoma: A review of literature. Clin Exp Metastasis 28: 493-503, 2011.

6. Sundaram MV: Canonical RTK-Ras-ERK signaling and related alternative pathways. WormBook 1: 1-38, 2013.

7. Jiménez G, Shvartsman SY and Paroush Z: The Capicua repressor-a general sensor of RTK signaling in development and disease. J Cell Sci 125: 1383-1391, 2012

8. Batchu SN and Korshunov VA: Novel tyrosine kinase signaling pathways: Implications in vascular remodeling. Curr Opin Nephrol Hypertens 21: 122-127, 2012.

9. Katoh M: WNT signaling pathway and stem cell signaling network. Clin Cancer Res 13: 4042-4045, 2007.

10. Green JL, Kuntz SG and Sternberg PW: Ror receptor tyrosine kinases: Orphans no more. Trends Cell Biol 18: 536-544, 2008.

11. Liu Y, Bhat RA, Seestaller-Wehr LM, Fukayama S, Mangine A, Moran RA, Komm BS, Bodine PV and Billiard J: The orphan receptor tyrosine kinase Ror2 promotes osteoblast differentiation and enhances ex vivo bone formation. Mol Endocrinol 21: 376-387, 2007

12. DeChiara TM, Kimble RB, Poueymirou WT, Rojas J, Masiakowski P, Valenzuela DM and Yancopoulos GD: Ror2, encoding a receptor-like tyrosine kinase, is required for cartilage and growth plate development. Nat Genet 24: 271-274, 2000.

13. Mehawej C, Chouery E, Maalouf D, Baujat G, Le Merrer M, Cormier-Daire V and Mégarbané A: Identification of a novel causative mutation in the ROR 2 gene in a Lebanese family with a mild form of recessive Robinow syndrome. Eur J Med Genet 55: 103-108, 2012.

14. Debebe Z and Rathmell WK: Ror2 as a therapeutic target in cancer. Pharmacol Ther 150: 143-148, 2015.

15. Rebagay G, Yan S, Liu C and Cheung NK: ROR1 and ROR2 in human malignancies: Potentials for targeted therapy. Front Oncol 2: 34, 2012.

16. Li L, Ying J, Tong X, Zhong L, Su X, Xiang T, Shu X, Rong R, Xiong L, Li H, et al: Epigenetic identification of receptor tyrosine kinase-like orphan receptor 2 as a functional tumor suppressor inhibiting $\beta$-catenin and AKT signaling but frequently methylated in common carcinomas. Cell Mol Life Sci 71: 2179-2192, 2014.

17. Lu BJ, Wang YQ, Wei XJ, Rong LQ, Wei D, Yan CM, Wang DJ and Sun JY: Expression of WNT-5a and ROR2 correlates with disease severity in osteosarcoma. Mol Med Rep 5: 1033-1036, 2012.

18. Ren D, Minami Y and Nishita M: Critical role of Wnt5a-Ror2 signaling in motility and invasiveness of carcinoma cells following Snail-mediated epithelial-mesenchymal transition. Genes Cells 16: 304-315, 2011

19. Enomoto M, Hayakawa S, Itsukushima S, Ren DY, Matsuo M Tamada K, Oneyama C, Okada M, Takumi T, Nishita M and Minami Y: Autonomous regulation of osteosarcoma cell invasiveness by Wnt5a/Ror2 signaling. Oncogene 28: 3197-3208, 2009.

20. Stricker S and Mundlos S: FGF and ROR2 receptor tyrosine kinase signaling in human skeletal development. Curr Top Dev Biol 97: 179-206, 2011.

21. Yamagata K, Li X, Ikegaki S, Oneyama C, Okada M, Nishita M and Minami Y: Dissection of Wnt5a-Ror2 signaling leading to matrix metalloproteinase (MMP-13) expression. J Biol Chem 287: 1588-1599, 2012.

22. Chang HR, Lian JD, Lo CW, Chang YC, Yang MY and Wang CJ: Induction of urothelial proliferation in rats by aristolochic acid through cell cycle progression via activation of cyclin D1/cdk4 and cyclin E/cdk2. Food Chem Toxicol 44: 28-35, 2006.

23. Li C, Chen H, Hu L, Xing Y, Sasaki T, Villosis MF, Li J, Nishita M, Minami Y and Minoo P: Ror2 modulates the canonical Wnt signaling in lung epithelial cells through cooperation with Fzd2. BMC Mol Biol 9: 11, 2008.

24. Lerner UH and Ohlsson C: The WNT system: Background and its role in bone. J Intern Med 277: 630-649, 2015.

25. Anastas JN and Moon RT: WNT signalling pathways as therapeutic targets in cancer. Nat Rev Cancer 13: 11-26, 2013.

26. Ford CE, Qian Ma SS, Quadir A and Ward RL: The dual role of the novel Wnt receptor tyrosine kinase, ROR2, in human carcinogenesis. Int J Cancer 133: 779-787, 2013. 
27. Wright TM, Brannon AR, Gordan JD, Mikels AJ, Mitchell C, Chen S, Espinosa I, van de Rijn M, Pruthi R, Wallen E, et al: Ror2, a developmentally regulated kinase, promotes tumor growth potential in renal cell carcinoma. Oncogene 28: 2513-2523, 2009.

28. Geng M, Cao YC, Chen YJ, Jiang H, Bi LQ and Liu XH: Loss of Wnt5a and Ror2 protein in hepatocellular carcinoma associated with poor prognosis. World J Gastroenterol 18: 1328-1338, 2012.

29. O'Connell MP, Fiori JL, Xu M, Carter AD, Frank BP, Camilli TC, French AD, Dissanayake SK, Indig FE, Bernier M, et al: The orphan tyrosine kinase receptor, ROR2, mediates Wnt5A signaling in metastatic melanoma. Oncogene 29: 34-44, 2010.

30. Wright TM, Brannon AR, Gordan JD, Mikels AJ, Mitchell C, Chen S, Espinosa I, van de Rijn M, Pruthi R, Wallen E, et al: Ror2, a developmentally regulated kinase, promotes tumor growth potential in renal cell carcinoma. Oncogene 28: 2513-2523, 2009.
31. Kobayashi M, Shibuya Y, Takeuchi J, Murata M, Suzuki H, Yokoo S, Umeda M, Minami Y and Komori T: Ror2 expression in squamous cell carcinoma and epithelial dysplasia of the oral cavity. Oral Surg Oral Med Oral Pathol Oral Radiol Endod 107: 398-406, 2009.

32. Morioka K, Tanikawa C, Ochi K, Daigo Y, Katagiri T, Kawano H, Kawaguchi H, Myoui A, Yoshikawa H, Naka N, et al: Orphan receptor tyrosine kinase ROR2 as a potential therapeutic target for osteosarcoma. Cancer Sci 100: 1227-1233, 2009.

33. Cheng BQ, Jiang Y, Zhu Q and Lin WG: Wnt $/ \beta$-catenin aids in regulating the proliferation of hepG2 cells mediated by thy-1. Genet Mol Res 13: 5115-5127, 2014. 